\title{
A First Search for Rapid Variations of Stellar and Circumstellar Balmer Discontinuity in Be Stars
}

S. Štefl

\author{
Astronomical Institute, Academy of Sciences, Ondřejov, Czech Republic \\ D. Baade
}

European Southern Observatory, Garching bei München, Germany

J. Cuypers

Belgian Royal Observatory, Brussel, Belgium

\begin{abstract}
Spectrophotometric observations of the Be star $\eta$ Cen obtained in May, 1993 show smooth variations of both the stellar and circumstellar Balmer discontinuity (BD) on a time scale of hours. Simultaneous photometry and high-resolution spectroscopy suggest possible correlations with the large-amplitude brightness and line-profile variations.
\end{abstract}

\section{Introduction}

Simultaneous monitoring of the southern Be star $\eta$ Cen by means of flux calibrated spectra $(\lambda \lambda 340-510 \mathrm{~nm}$, resolving power 5,000, temporal sampling 35 minutes), high-resolution line profiles (Si III $\lambda 455.3$ and $456.2 \mathrm{~nm}$, resolving power 65,000 , temporal sampling 15-20 minutes) and uvby magnitudes was carried out with three telescopes of the La Silla Observatory. The aim was to search for the rapid BD variations predicted by Štefl et al. (Štefl, S., Baade D., Harmanec, P., Balona L.A. 1995, A\&A, 294, 135), and to investigate their correlations, if any, with the known rapid light and line-profile variations. Due to exceptionally bad weather, good quality data were obtained during one night only (May 10/11, 1993). The new results reported here are therefore preliminary.

\section{Results}

The BCD parameters $D$ and $d$ (height of the photospheric and circumstellar $\mathrm{BD}$, respectively, measured at the common wavelength $\lambda_{0}=370 \mathrm{~nm}$; for the definition see, e.g., Divan L.: 1979, IAU Coll. 47, p. 247 and references therein) characterizing the Balmer discontinuity were measured. The main results are:

- Variations of the photospheric BD were detected at high confidence level (peak-to-peak amplitude $4 \%$ of the ambient continuum flux).

- Small variations ( $1 \%$ amplitude) of the circumstellar BD (in emission) were also found. 
- Scattered observations from two more nights show that the variations of the BD parameters probably follow the 0.6424-day photometric period.

The range in $u-b$ amounts to $0 ?^{m} 02$. However, unlike 1992 when the peak-topeak amplitude was $0 r^{m} 03$ (Stefl et al. 1995), the 1993 observations do not cover the phases of light and $u-b$ colour maximum. Therefore, the true amplitudes of the BD variations may be larger than observed so far.

Ultra-rapid light variations observed after phase $0{ }^{P} 5$ may represent an intrinsic flickering. No such flickering was observed in 1992 (Štefl et al. 1995).

Almost no Si III line-profile variations were detected in the first half of the night. Profiles with large asymmetric wings extending to more than $-1000 \mathrm{~km} / \mathrm{s}$ appear in the phase interval $0 . P 5-0 . P 6$ and were followed by strong absorption bumps arising in the far violet wings and moving to the line centre.

Two components can be distinguished in the variability of the $1^{\text {st }}$ moment of the Si III 455.3 line: i) the smooth (probably phase dependent) variations described by Štefl et al. (1995), ii) an abrupt decrease during the phase interval $0{ }^{P} 45-0 . P 60$ when the extended violet wings were present.

If fixed phase relations between the rapid variations of the 3 quantities studied (Balmer discontinuity, line profiles, and light and colour) are assumed, our observations suggest the following correlations:

- The photospheric BD height may correlate with brightness and low-order line-profile variations which maintain a stable period of 0.6424 days.

- The variability of the circumstellar BD coincides with the occurrence of rapid photometric flickering and of strong narrow features moving across the Si III line profiles. The two high-frequency phenomena appear when the circumstellar Balmer height $d$ is low.

\section{Discussion}

$\mathrm{BD}$ variations are detectable from ground-based data. Our observations of $\eta$ Cen represent the first direct spectroscopic evidence of rapid BD variations in earlytype stars. Possibly, not only the stellar Balmer discontinuity but the circumstellar one as well varies with phase of the general photospheric variability.

The sensitivity of height and position of the BD to temperature and density, respectively, and possible phase relations between variations of $\mathrm{BD}$, line profiles, and light and colour may permit, for the first time, an analysis of rapid variations to be performed separately for photosphere and circumstellar envelope.

The narrow moving features in the Si III lines, photometric flickering, and the low state of the circumstellar Balmer jump may all be connected with processes in the circumstellar gas. We note that these phenomena were observed shortly after the development of the highly asymmetric Si III profiles which persisted for less than 1 hour. If the latter are due to mass outflow and we may assume an expansion velocity of $1000 \mathrm{~km} / \mathrm{s}$ and $R_{\star}=4.0 R_{\odot}$ (Štefl et al. 1995), the processes giving rise to these rapid variations would take place within about $1 R_{\star}$ of the photosphere.

Acknowledgments. The authors thank Dr. J. Zorec for constructive comments to the manuscript. 